\title{
Low Impact of Urinary Cortisol in the Assessment of Hydrocortisone Replacement Therapy
}

Authors

Affiliations

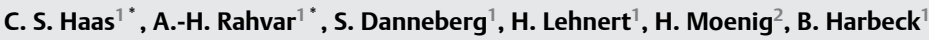

Department of Medicine I, University of Luebeck, Luebeck, Germany

Department of Medicine I, University of Kiel, Kiel, Germany
Key words

- urinary cortisol

- adrenal insufficiency

- hydrocortisone replacement therapy received 08.03 .2016 accepted 09.05.2016

Bibliography DOI http://dx.doi.org/ 10.1055/s-0042-108729 Published online: June 14, 2016 Horm Metab Res 2016; 48: $571-574$ (c) Georg Thieme Verlag KG Stuttgart · New York ISSN 0018-5043

Correspondence

\section{B. Harbeck, MD}

Department of Medicine I University of Luebeck Ratzeburger Allee 160 23538 Luebeck Germany Tel.: + 49/451/500 6406 Fax: $+49 / 451 / 5006022$ birgit.harbeck@uksh.de

\section{Abstract}

$\nabla$

Hydrocortisone replacement therapy is a cornerstone in the treatment of adrenal insufficiency (AI). While urinary cortisol has been used as a diagnostic tool for AI, it remains unclear whether it is a useful parameter to monitor hydrocortisone replacement therapy. Aim of this study was to evaluate possible differences in cortisol metabolism between adrenal insufficient patients and healthy subjects and to assess the value of urinary cortisol in AI management. In a case-control study, urinary cortisol excretion was determined in 14 patients with primary and secondary $\mathrm{AI}$ receiving hydrocortisone infusions from midnight to 8:00 AM. Results were correlated with serum cortisol levels and compared to urinary values obtained from 53 healthy volunteers. Urinary cortisol excretion in healthy subjects was $14.0 \pm 7.8 \mu \mathrm{g} / 8 \mathrm{~h}$ (range: $0.24-35.4$ ), levels did not

\section{Introduction}

Patients with adrenal insufficiency (AI) require life-long cortisol replacement therapy usually consisting of 2 or 3 hydrocortisone doses, the last dose in mid-afternoon or early evening. These treatment regimens, however, fail to mimic the circadian rhythm of cortisol secretion. Due to the short half-life of hydrocortisone, cortisol levels during the night are invariably low, whereas they are found to be supraphysiological $2 \mathrm{~h}$ after ingestion of hydrocortisone [1-3]. The consequences of nocturnal cortisol deficiency as well as those of temporary hypercortisolism are yet unknown [4]; however, inadequate treatment with short-acting glucocorticoids may result in adrenal crisis [5]. It has also been suggested that inadequate levels of cortisol in hydrocortisone

${ }^{*}$ These authors contributed equally to this work differ between 3 groups aged 20-34 years, 35-49 years, and $\geq 50$ years. Patients with AI receiving hydrocortisone infusions demonstrated significantly higher rates of urinary cortisol excretion (51.6 $\pm 37.8 \mu \mathrm{g} / 8 \mathrm{~h}$; range 17.1-120.0, $\mathrm{p}<0.001)$; the values correlated with serum cortisol levels $\left(\mathrm{r}^{2}=0.98\right)$. Of interest, patients with secondary AI showed significantly higher serum cortisol levels after hydrocortisone infusion than those with primary AI, conceivably due to residual adrenal function. In conclusion, we showed that: (i) there is a wide inter-individual variability in urinary cortisol excretion rates; (ii) cortisol metabolism in adrenal insufficient patients differs when compared to controls; (iii) there is a strong correlation between urinary and serum cortisol levels; and (iv) urinary cortisol levels despite their variability may help to discriminate between secondary and primary adrenal insufficiency.

replacement therapy (HRT) lead to a higher prevalence of cardiovascular disease and diabetes mellitus and affect quality of life [6,7]. Therefore, surveillance of cortisol replacement therapy is essential to avoid serious side effects. Due to the pharmacokinetic structure of oral hydrocortisone, biochemical analyses of serum cortisol are of no use and surveillance should be achieved through clinical monitoring [8]. Recently, several studies used urinary corticosteroid profiles to monitor replacement doses in patients with adrenal hypofunction $[9,10]$. However, a possible difference in the metabolism of cortisol between healthy subjects and patients with primary or secondary AI may confound interpretation. In fact, little information is available whether urinary excretion of cortisol is a useful alternative to assess the quality of HRT. Aim of the present study was to perform a quantitative analysis of urinary cortisol excretion of affected AI patients compared to healthy subjects. 


\section{Materials and Methods}

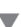

Fourteen patients with primary or secondary AI (11 women, 3 men, age 29-70 years) received hydrocortisone infusion between midnight and 08:00 AM at a dose according to AlShoumer et al. [11], in order to mimic the physiological rise in healthy subjects ( $\bullet$ Table 1 for hydrocortisone infusion rates) and to identify possible differences in cortisol metabolism between AI patients and controls. Blood for serum cortisol analysis was drawn at 08:00 AM. The study was approved by the Ethics committee at the University of Kiel (AZ D 424/03) and informed consent was obtained from each subject.

Urinary samples were analysed for cortisol excretion levels by using a commercial immunoassay (Immulite 2000, Siemens Healthcare Diagnostics, Deerfield, IL, USA). Healthy volunteers without clinical signs of hypercortisolism collected their urine from 00:00 to 08:00 AM to serve as controls. A history of pathological renal or liver test results was an exclusion criterion, and all participants of the control group were reported to have no prior existing endocrine disorder. The volunteers were divided into 3 subgroups depending on age: 20-34 years, 35-49 years,

Table 1 Hydrocortisone infusion rate.

\begin{tabular}{|l|l|}
\hline Time & Hydrocortisone dose $(\boldsymbol{\mu g} / \mathbf{k g} / \mathbf{m i n})$ \\
\hline 00:00-01:00 AM & 0.15 \\
\hline 01:00-02:00 AM & 0.15 \\
\hline 02:00-03:00 AM & 0.10 \\
\hline 03:00-04:00 AM & 0.20 \\
\hline 04:00-05:00 AM & 0.20 \\
\hline 05:00-06:00 AM & 0.80 \\
\hline 06:00-07:00 AM & 0.90 \\
\hline 07:00-08:00 AM & 0.63 \\
\hline
\end{tabular}

and at least 50 years old. In AI patients, values urinary and blood cortisol were comparatively analysed. Values of urinary cortisol excretion are depicted as mean \pm standard deviation as well as range per group, and were compared between groups using student's $t$-test. A p-value $<0.05$ was considered significant. Fisher's exact test was used to compare categorical data. To exclude a potential role of renal function on urinary cortisol excretion, serum creatinine values of Al patients were measured with subsequent calculation of the glomerular filtration rate (eGFR) using the CKDEpi formula that incorporates age and sex. Pearson's correlation coefficient was determined to assess the relationship between serum and urinary cortisol levels in AI patients as well as between age or glomerular function and urinary cortisol excretion.

\section{Results \\ $\nabla$}

A total of $14 \mathrm{AI}$ patients and 53 healthy volunteers were included in the study. Both groups were comparable with respect to age (51.1 \pm 12.3 vs. $43.1 \pm 14.3$ years), body weight $(74.8 \pm 13.3$ vs. $73.3 \pm 15.0 \mathrm{~kg})$, and BMI $\left(26.5 \pm 4.9\right.$ vs. $\left.24.2 \pm 3.7 \mathrm{~kg} / \mathrm{m}^{2}\right)$. Median age of primary and secondary AI patients was 54 vs. 43 years $(p=0.27)$; body weight was not significantly different ( 78.5 vs. $73.7 \mathrm{~kg} ; \mathrm{p}=0.77$ ).

Healthy volunteers showed a urinary cortisol excretion rate of $14.0 \pm 7.8 \mu \mathrm{g} / 8 \mathrm{~h}$ with a wide inter-individual range (0.24-35.4). There was no significant difference in cortisol excretion rates between the healthy subgroups of subjects ( $\bullet$ Table 2 ). In the subgroup of healthy volunteers between 20 and 34 years $(n=19$, 13 females), the mean cortisol urinary excretion rate was $13.1 \pm 6.4 \mu \mathrm{g} / 8 \mathrm{~h}$; in the subgroup of volunteers between 35 and 49 years $(\mathrm{n}=16,9$ females $) 16.2 \pm 9.1 \mu \mathrm{g} / 8 \mathrm{~h}$ and in the group $>50$ years $(\mathrm{n}=18,9$ females), the mean rate was $12.8 \pm 7.5 \mu \mathrm{g} / 8 \mathrm{~h}$.

Table 2 Urinary cortisol excretion rates ( $8 \mathrm{~h}$ ) and serum cortisol levels in healthy subjects and patients with primary or secondary adrenal insufficiency.

\begin{tabular}{|c|c|c|c|c|c|c|c|}
\hline \multirow[b]{2}{*}{ Subject } & \multicolumn{3}{|c|}{$\begin{array}{l}\text { Healthy subjects } \\
\text { Urinary cortisol excretion rate }[\mu \mathrm{g} / 8 \mathrm{~h}] \\
\ldots \text { under normal conditions }\end{array}$} & \multicolumn{2}{|c|}{$\begin{array}{l}\text { Adrenal insufficiency } \\
\text { Urinary cortisol excretion rate }[\mu \mathrm{g} / 8 \mathrm{~h}] \\
\ldots \text { following } \mathrm{HC} \text { infusion }\end{array}$} & \multicolumn{2}{|c|}{$\begin{array}{l}\text { Serum cortisol level } \\
{[\mu \mathrm{g} / \mathrm{dl}]}\end{array}$} \\
\hline & $20-34$ years & $35-50$ years & $>50$ years & Primary & Secondary & Primary & Secondary \\
\hline 1 & 2.6 & 0.24 & 3.3 & 17.1 & 25.8 & 6.7 & 9.9 \\
\hline 2 & 3.4 & 4.3 & 4.9 & 21.7 & 39.0 & 10.3 & 17.7 \\
\hline 3 & 6.1 & 6.7 & 6.0 & 31.8 & 42.0 & 12.3 & 19.4 \\
\hline 4 & 6.5 & 8.6 & 6.8 & 35.7 & 53.4 & 14.8 & 20.6 \\
\hline 5 & 8.0 & 10.8 & 7.4 & 42.6 & 64.8 & 17.0 & 21.4 \\
\hline 6 & 9.4 & 10.8 & 7.6 & 64.0 & 70.4 & 17.9 & 22.0 \\
\hline 7 & 9.6 & 11.5 & 9.1 & & 93.6 & & 29.5 \\
\hline 8 & 10.0 & 14.7 & 11.1 & & 120.0 & & 40.3 \\
\hline 9 & 10.2 & 18.2 & 11.5 & & & & \\
\hline 10 & 12.6 & 19.2 & 12.0 & & & & \\
\hline 11 & 15.5 & 19.3 & 12.6 & & & & \\
\hline 12 & 15.5 & 19.8 & 13.8 & & & & \\
\hline 13 & 15.7 & 25.0 & 15.0 & & & & \\
\hline 14 & 17.0 & 28.6 & 15.0 & & & & \\
\hline 15 & 18.4 & 30.7 & 16.1 & & & & \\
\hline 16 & 21.0 & 31.0 & 17.9 & & & & \\
\hline 17 & 21.5 & & 25.2 & & & & \\
\hline 18 & 22.5 & & 35.4 & & & & \\
\hline 19 & 24.0 & & & & & & \\
\hline Mean & 13.1 & 16.2 & 12.8 & 35.5 & 63.6 & 13.2 & 22.6 \\
\hline SD & 6.4 & 9.1 & 7.5 & 15.3 & 29.0 & 3.9 & 8.4 \\
\hline Mean & & 14.0 & & & & & \\
\hline$S D$ & & 7.8 & & & & & \\
\hline
\end{tabular}


Although the fraction of women in the AI group was higher than in all controls, the difference was not significant (58 vs. $79 \%$, $\mathrm{p}=0.32$ ). In the AI patients, age was comparable (median 51 vs. 51 years, $p=0.65$ ), with 3 male participants in the secondary AI group. AI patients receiving hydrocortisone infusions showed significantly higher urinary cortisol excretion rates than healthy controls (51.6 $\pm 27.8 \mu \mathrm{g} / 8 \mathrm{~h}$; range: $17.1-120.0 ; \mathrm{p}<0.0001$; $\odot$ Table 2 ). Of note, patients with secondary AI $(n=8)$ showed significantly higher serum cortisol levels and urinary cortisol excretion rates after hydrocortisone infusion than the group with primary AI $(\mathrm{n}=6 ; \mathrm{p}<0.05 ; \odot$ Table 2$)$. Urinary values clearly correlated with serum cortisol levels in patients with both primary and secondary AI $\left(r^{2}=0.98\right.$, $\odot$ Fig. 1a). Urinary cortisol excretion rates in both groups of healthy volunteers and AI patients showed no significant correlation regarding age ( $\Theta$ Fig. 1b) and body weight $\left(r^{2}=0.20\right.$, data not shown). Similarly, renal function in the AI patients (mean creatinine $0.78 \pm 0.15 \mathrm{mg} / \mathrm{dl}$, range: $0.6-1.06$ ) did not correlate with urinary cortisol excretion $(\bullet$ Fig. 1c). There was no significant difference in eGFR between both groups with primary and secondary AI $\left(100.5 \pm 8.7 \mathrm{ml} / \mathrm{min} / 1.73 \mathrm{~m}^{2}\right.$ vs. $\left.90.3 \pm 18.9 \mathrm{ml} / \mathrm{min} / 1.73 \mathrm{~m}^{2}, \mathrm{p}=0.28\right)$.

\section{Discussion}

$\nabla$

Urinary cortisol excretion over time has been considered a potential tool in diagnosing and monitoring AI patients with HRT. Since little is known under normal conditions, we determined the excretory rate in healthy subjects over the first $8 \mathrm{~h}$ of a normal work day. In addition, we used a continuous hydrocortisone infusion in patients with $\mathrm{AI}$ in order to mimic the physiological biorhythm during this timeframe, as previously described [11]. Furthermore, we intended to determine possible differences in cortisol metabolism between healthy subjects and in patients with primary and secondary AI. Jung et al. found that total plasma cortisol correlates significantly with urinary cortisol after intravenous hydrocortisone application [10]. In the present study, we could confirm these findings in AI patients receiving hydrocortisone infusions. Interestingly, there was a strong inter-individual variability of cortisol excretion levels despite using a uniform weight-adapted hydrocortisone infusion rate. This may point to a limited diagnostic value of urinary cortisol measurement despite its wide use in clinical practice. Recently, Raff and colleagues addressed this variability in urine cortisol levels and discussed the controversies of using urine free cortisol in the diagnosis of Cushing's syndrome [12]. The most intriguing finding in the present study, however, is a difference between the AI subgroups: Values for serum and urinary cortisol were significantly higher in patients with secondary compared to primary AI. Interestingly, the infusion doses used seemed to be excessive in almost half of the patients with secondary AI. It is possible that endogenous secretion of cortisol in the AI patients may have an effect on the observed variants of urinary and serum cortisol levels. Thus, urinary cortisol levels despite their variability may help to identify residual adrenal function in AI. In addition, these data suggest that AI patients may require an individualised dose of hydrocortisone. Normally, the majority of circulating cortisol is bound to CBG and albumin with less than $5 \%$ of circulating cortisol as the physiologically active being free. This unbound cortisol is filtered by the renal glomerulus and hereafter excreted in the urine. CBG is mostly produced by the liver, upregulated by estrogens, and suppressed

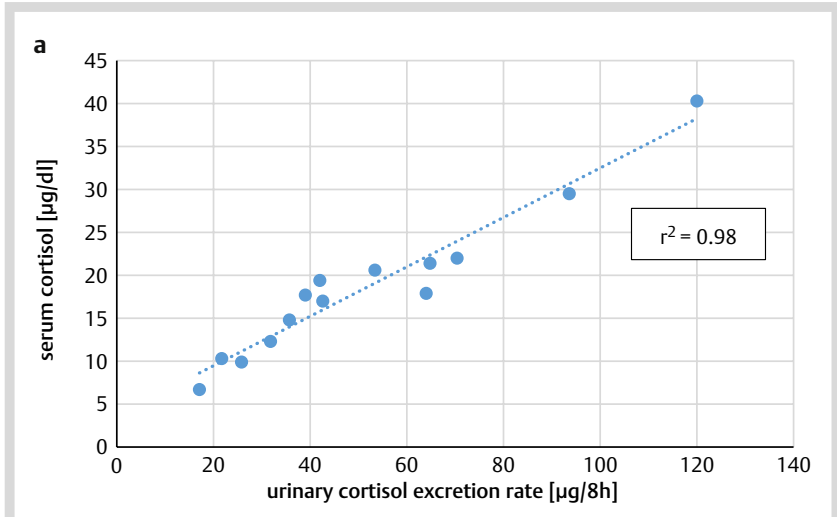

b
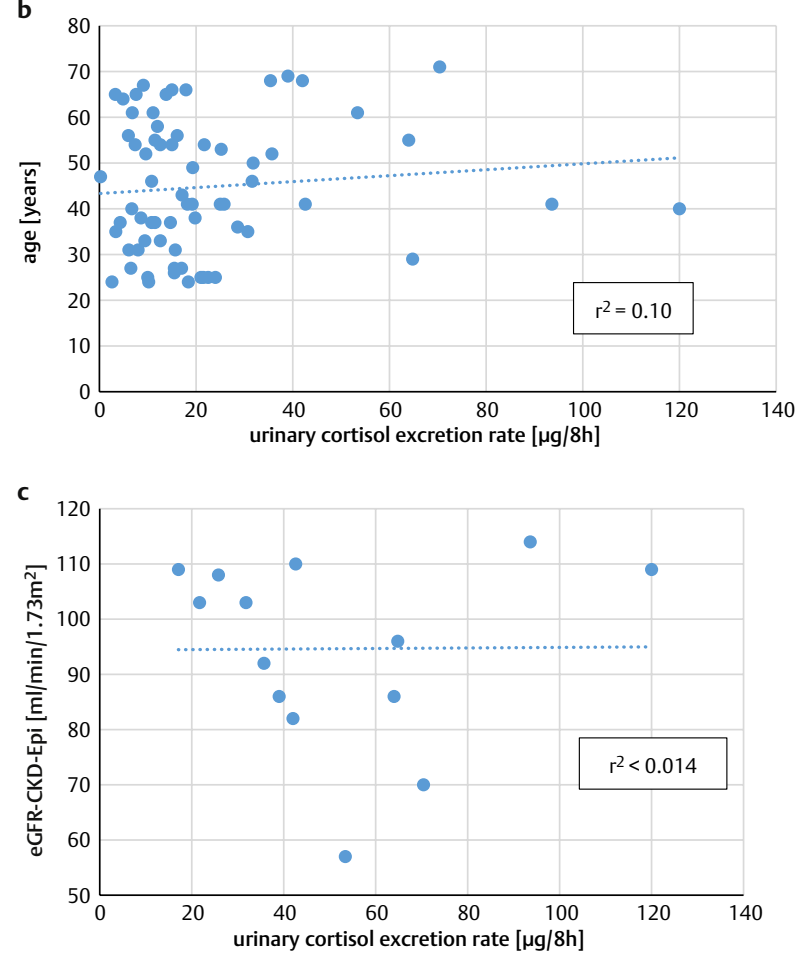

Fig. 1 a Patients with adrenal insufficiency (Al) receiving hydrocortisone infusions showed urinary cortisol values that clearly correlated with serum cortisol levels. $\mathbf{b}$ In both groups, patients with Al as well as healthy volunteers, there was no significant correlation between urinary excretion of cortisol and age. c Urinary cortisol values in Al patients were independent from renal excretory function, depicted as estimated glomerular filtration rate that was determined by the CKD-Epi formula (eGFR-CKD-Epi). (Color figure available online only).

by steroids with high levels observed in pregnancy and low levels in patients with cirrhosis $[13,14]$. Since we only included healthy patients as controls, liver function was not considered to have an effect on cortisol levels. Although also oral contraceptives may have had an effect on urinary cortisol levels in the AI patients, this is unlikely because age was comparable between both groups and the 3 males were in the secondary AI where urinary cortisol levels were significantly higher.

While the present data point to a possible weak relationship between urinary cortisol and age, Ragnarsson and co-workers recently demonstrated in a cross-sectional study including a population sample of 348 persons aged 38-77 years that urinary free cortisol rather decreased in the elderly [15]. The number and sort of individuals included in our study is different though so that the results may not be comparable. Previous studies have 
shown that glomerular filtration rate and the amount of fluid intake may have an effect on urinary cortisol excretion rates $[16,17]$. However, we did not see a statistically significant correlation between renal function and urinary cortisol excretion rates in our AI patients receiving hydrocortisone infusions. There were also no differences with respect to glomerular filtration rate when patients with primary and secondary AI were compared. This may be due to the fact that renal function in AI patients was not seriously impaired as demonstrated by normal or only slightly elevated serum creatinine levels but may be distinct in patients with more pronounced renal insufficiency.

It has been shown that urinary cortisol excretion is dependent on the corticosteroid-binding globulin capacity. Intravenous and oral hydrocortisone substitution exceeds the binding capacity of corticosteroid globulin, temporarily leading to higher cortisol excretion rates in patients receiving hydrocortisone replacement therapy. A possible residual adrenal function in patients with secondary AI may lead to a faster saturation of CBG capacity resulting in significantly higher urinary cortisol excretion rates in these patients. This has also been described in patients suffering from Cushing's disease, where supraphysiological levels of cortisol are a result of the pathophysiological mechanism [18]. Although measuring urinary cortisol excretion allows to determine endogenous cortisol production, its use as a monitoring instrument may not be conceivable. Highly variable levels of serum cortisol in hydrocortisone replacement therapy render the monitoring of AI difficult. Other factors like liver disease, nephrotic syndrome, and oral estrogens must also be taken into account since they influence the amount of corticosteroid binding globulin $[19,20]$. Finally, different types of assays are used to assess urinary cortisol excretion. These assays can be antibodybased or structurally-based, leading to varying reference values and different levels of specificity and sensitivity [21].

The present study harbors some strengths and limitations related to the study design. The healthy control group was large enough to determine physiological urinary cortisol excretion. In addition, the setting of hydrocortisone infusions in AI patients within the first $8 \mathrm{~h}$ of a day is ideal to mimic the physiological peak of endogenous cortisol production in the morning. Contrary to Al-Shoumer et al. [11], whose study design was limited to hypopituitary adults, the group of AI patients investigated here was somewhat heterogeneous with regard to the underlying diseases; for example, patients with pituitary diseases often have an ACTHindependent residual secretion of cortisol, which may have influenced some results. However, there was a wide range of values possibly in part due to the small sample size in this pilot study. In conclusion, we showed that: (i) urinary cortisol excretion rates vary strongly in healthy subjects and patients with AI; (ii) cortisol metabolism in AI patients is different compared to controls; (iii) urinary cortisol correlates well with serum cortisol in patients with AI; and (iv) patients with secondary AI demonstrate significantly higher urinary and serum cortisol levels following HC infusions than those with primary AI. Presumably, urinary cortisol may be helpful in the assessment of residual adrenal function in patients with $\mathrm{AI}$ rather than to be useful as a monitoring parameter for HRT.

\section{Conflict of Interest}

$\nabla$

None of the authors have any potential conflicts of interest associated with this research.

\section{References}

1 Howlett TA. Adrenocorticotrophin deficiency: assessment and monitoring of glucocorticoid replacment. In: The Diagnosis and Treatment of Pituitary Insufficiency. Lamberts SWJ. (ed.). Bristol: BioScientifica; 1997: 81-90

2 De Vile CJ, Stanhope R. Hydrocortisone replacement therapy in children and adolescents with hypopituitarism. Clin Endocrinol (Oxford) 1997; 47: 37-41

3 Groves RW, Toms GC, Houghton BJ, Monson JP. Corticosteroid replacement therapy: twice or thrice daily? J R Soc Med 1988; 81: 514-516

4 Harbeck B, Kropp P, Mönig H. Effects of Short-Term Nocturnal Cortisol Replacement on Cognitive Function and Quality of Life in Patients with Primary or Secondary Adrenal Insufficiency: A Pilot Study. Appl Psychophysiol Biofeedback 2009; 34: 113-119

5 Rushworth RL, Torpy DJ. Modern Hydrocortisone Replacement Regimens in Adrenal Insufficiency Patients and the Risk of Adrenal Crisis. Horm Metab Res 2015; 47: 637-642

6 Petersons CJ, Mangelsdorf BL, Thompson CH, Burt MG. Acute effect of increasing glucocorticoid replacement dose on cardiovascular risk and insulin sensitivity in patients with adrenocorticotrophin deficiency. J Clin Endocrinol Metab 2014; 99: 2269-2276

7 Ekman B, Alstrand N, Bachrach-Lindström M, Jenmalm MC, Wahlberg J. Altered chemokine Th1/Th2 balance in Addison's disease: relationship with hydrocortisone dosing and quality of life. Horm Metab Res 2014; 46: 48-53

8 Derendorf H, Möllmann H, Barth J, Möllmann C, Tunn S, Krieg M. Pharmacokinetics and oral bioavailability of hydrocortisone. J Clin Pharmacol 1991; 31: 473-476

9 Sherlock M, Behan LA, Hannon MJ, Alonso AA, Thompson CJ, Murray RD, Crabtree N, Hughes BA, Arlt W, Agha A, Toogood AA, Stewart PM. The modulation of corticosteroid metabolism by hydrocortisone therapy in patients with hypopituitarism increases tissue glucocorticoid exposure. Eur J Endocrinol 2015; 173: 583-593

10 Jung C, Greco S, Nguyen H, Ho JT, Lewis JG, Torpy DJ, Inder WJ. Plasma, salivary and urinary cortisol levels following physiological and stress doses of hydrocortisone in normal volunteers. BMC Endocr Disord 2014; 14: 91

11 Al-Shoumer KA, Anyaoku V, Ali K, Johnston DG. Metabolic effects of short-term reproduction of the normal diurnal cortisol rhythm in hypopituitary adults. Endocrinol Metabol 1997; 4: 207-214

12 Raff H, Auchus RJ, Findling JW, Nieman LK. Urine free cortisol in the diagnosis of Cushing's syndrome: is it worth doing and, if so, how? J Clin Endocrinol Metab 2015; 100: 395-397

13 Cizza G, Rother KI. Cortisol binding globulin: more than just a carrier? J Clin Endocrinol Metab 2012; 97: 77-80

14 Boisseau N, Enea C, Diaz V, Dugué B, Corcuff JB, Duclos M. Oral contraception but not menstrual cycle phase is associated with increased free cortisol levels and low hypothalamo-pituitary-adrenal axis reactivity. J Endocrinol Invest 2013; 36: 955-964

15 Ragnarsson 0 , Trimpou $P$, Oleröd $G$, Landin-Wilhelmsen $K$. The association between urinary cortisol excretion and cardiovascular risk factors, bone status and quality of life in the population. Steroids 2015; 101: 71-77

16 Chan KC, Lit LC, Law EL, Tai MH, Yung CU, Chan MH, Lam CW. Diminished urinary free cortisol excretion in patients with moderate and severe renal impairment. Clin Chem 2004; 50: 757-759

17 Mericq MV, Cutler GB Jr. High fluid intake increases urine free cortisol excretion in normal subjects. J Clin Endocrinol Metab 1998; 83: 682-684

18 Mengden T, Hubmann P, Müller J, Greminger P, Vetter W. Urinary free cortisol versus 17-hydroxycorticosteroids: a comparative study of their diagnostic value in Cushing's syndrome. Clin Investig 1992; 70: 545-548

19 Tan T, Chang L, Woodward A, McWhinney B, Galligan J, Macdonald GA, Cohen J, Venkatesh $B$. Characterising adrenal function using directly measured plasma free cortisol in stable severe liver disease. J Hepatol 2010; 53: 841-848

20 Klose M, Lange M, Rasmussen AK, Skakkebaek NE, Hilsted L, Haug E, Andersen $M$, Feldt-Rasmussen $U$. Factors influencing the adrenocorticotropin test: role of contemporary cortisol assays, body composition, and oral contraceptive agents. J Clin Endocrinol Metab 2007; 92: 1326-1333

21 Wood L, Ducroq DH, Fraser HL, Gillingwater S, Evans C, Pickett AJ, Rees $D W$, John $R$, Turkes $A$. Measurement of urinary free cortisol by tandem mass spectrometry and comparison with results obtained by gas chromatography-mass spectrometry and two commercial immunoassays. Ann Clin Biochem 2008; 45: 380-388 